\title{
Topological affine quadrangles
}

\author{
Nils Rosehr \\ Dedicated to Professor Hermann Hähl on the occasion of his $60^{\text {th }}$ birthday.
}

\begin{abstract}
The affine derivation of a generalized quadrangle is the geometry induced on the vertices at distance 3 or 4 of a given point. We characterize these geometries by a system of axioms which can be described as a modified axiom system for affine planes with an additional parallel relation and parallel axiom. A second equivalent description which makes it very easy to verify that, for example, ovoids and Laguerre planes yield generalized quadrangles is given. We introduce topological affine quadrangles by requiring the natural geometric operations to be continuous and characterize when these geometries have a completion to a compact generalized quadrangle. In the connected case it suffices to assume that the topological affine quadrangle is locally compact. Again this yields natural and easy proofs for the fact that many concrete generalized quadrangles such as those arising from compact Tits ovoids are compact topological quadrangles. In an appendix we give an outline of the theory of stable graphs which is fundamental to this work.
\end{abstract}

Keywords: generalized quadrangle, affine quadrangle, parallel axiom, topological geometry, completion

MSC 2000: 51E12, 51H10

\section{Introduction}

In [25] Jacques Tits introduced generalized polygons. These objects can be regarded as graphs or a as incidence geometries. A graph $(V, E)$ is just a set $V$, the vertex set, together with a set $E$ of subsets of $V$ with precisely two elements, the edge set. The graph $(V, E)$ is called bipartite if it has a part, which is a subset $P \subseteq V$ of the vertex set such that all edges of $(V, E)$ contain one vertex from $P$ 



\section{Affine quadrangles}

Affine quadrangles were defined by Pralle and by B. Stroppel. Pralle's aim was to give a characterization (in terms of axioms) for the geometry obtained by removing a geometric hyperplane from a generalized quadrangle; see [14]. There are three types of geometric hyperplanes. We are only interested in the case where this hyperplane is a point star; i.e. the set of points at distance 0 or 2 from a given point. Here only structures of this kind will be called affine quadrangles, because the analogy to affine planes is only given in this case. Stroppel gives another axiom system for this type; see [24]. She calls her objects pointaffine quadrangles. The two relations used in her description are basically our two parallel relations, whereas our axiom system is somewhat closer to Pralle's. It stresses the analogy to affine planes and makes it straightforward to define topological affine quadrangles.

Definition 2.1. Let $\mathcal{A}=(P, L, I)$ be an incidence geometry, and define the relations

$$
\begin{aligned}
& g \mid h: \Longleftrightarrow \forall a \in D_{1}(g), b \in D_{1}(h): d(a, h)=d(b, g) \quad \text { and } \\
& g \| h: \Longleftrightarrow d(g, h) \in\{0,6\}
\end{aligned}
$$

for $g, h \in L$. The relations | and $\|$ are regarded as subsets of $L^{2}$ and we set $\nmid:=L^{2} \backslash \mid$ and $\nVdash:=L^{2} \backslash \|$. We call $\|$ strong parallelism and | weak parallelism or simply parallelism. The incidence geometry $\mathcal{A}$ is called an affine quadrangle, if the following axioms (A1) to (A4) hold.

(A1) Some point row has at least 2 and some line pencil at least 3 elements.

(A2) The girth of $\mathcal{A}$ is greater than 6 , and we have $d(L \times L) \leq 6$.

(A3) For every $(p, h) \in P \times L$ there is a unique $l \in D_{1}(p)$ such that $l \mid h$.

(A4) For every $(g, h) \in \nmid$ there is a unique $l \in D_{2}(g)$ such that $l \| h$.

For later use we record a significant weakening of (A2).

(A2') The girth of $\mathcal{A}$ is greater than 4; i.e. there are no digons.

Note the similarity between (A3) and (A4) and that (A3) is just the parallel axiom for affine planes. The motivation of the above definition is given by the following observation, which is an easy consequence of the definition of generalized quadrangles. 

Then $\overline{\mathcal{A}}:=(\bar{P}, \bar{L}, \bar{I})$ is a generalized quadrangle, and the derivation at $\infty$ satisfies $\overline{\mathcal{A}}_{\infty}=\mathcal{A}$. If $Q$ is a generalized quadrangle and $p$ a point of $Q$, then $\overline{Q_{p}}$ is isomorphic to $Q$.

Proof. We will use the symbol $d$ for the distance function in $\mathcal{A}$ and begin with collecting some facts about $\mathcal{A}$.

(a) The relation $\|$ is contained in $\mid$ : If there were lines $g$ and $h$ with $g \| h$ and $g \nmid h$, then by (A4) there would be a line $g^{\prime} \in D_{2}(g)$ with $g^{\prime} \| h$, and we would have $g^{\prime} \| g$. If $g \wedge g^{\prime} \in D_{1}(h)$ this leads to the contradiction $g=$ $u(h, g)=g^{\prime}$ by the uniqueness part from (A4) and otherwise to the contradiction $g=\lambda\left(g \wedge g^{\prime}, h\right)=g^{\prime}$ by the uniqueness part from (A5).

(b) If we have $\left|D_{1}(g)\right| \geq 2$ and $g \nmid h$ for lines $g$ and $h$, then $\left|D_{1}(h)\right| \geq 2$ and $d(g, h) \leq 4:$ If $d(g, h)=2$, we can choose by (A1) and (A3) a line $g^{\prime} \in D_{2}(g)$ not incident with $g \wedge h$ and not parallel to $h$. Then we have $u\left(h, g^{\prime}\right) \wedge h \neq g \wedge h$ by the uniqueness part from (A5). If $d(g, h)>2$, we apply (A5) to two distinct points incident with $g$ and to the line $h$. The lines obtained are distinct, and one of them must intersect $h$ by the uniqueness part from (A4). Thus $d(g, h)=4$, and the intersection point on $h$ is not equal to $u(h, g)$ by the uniqueness part from (A5).

(c) We have $d(p, h) \leq 5$ for $p \in P$ and $h \in L$ : By (A1) there is a line $g$ with at least two points on it. By (A1) and (A3) we can choose a line $l \in D_{1}(p)$ which is neither parallel to $g$ nor $h$. Now applying (b) to $g$ and $l$ as well as to $l$ and $h$ we obtain $d(l, h) \leq 4$ and therefore $d(p, l) \leq 5$.

We can now prove that $\overline{\mathcal{A}}$ is a generalized quadrangle. Let $p \in \bar{P}$ and $l \in \bar{L}$ with $(p, l) \notin \bar{I}$. We begin with showing the existence of joining paths $(p, m, q, l)$. We need to treat 3 times 2 cases two of which have two subcases; see (c). Here is a table for $(m, q)$ in all 8 cases; we write $m, q$ instead of $(m, q)$ to make the table more readable.

\begin{tabular}{l|ll|l} 
& \multicolumn{2}{|c|}{$l \in L$} & \multicolumn{1}{|c}{$l=[g]$} \\
\hline \multirow{2}{*}{$p \in P$} & $d(p, l)=3:$ & $\lambda(p, l), \pi(p, l)$ & $\iota(p, g), \llbracket \iota(p, g) \rrbracket$ \\
& $d(p, l)=5:$ & $\lambda(p, l), \llbracket \lambda(p, l) \rrbracket$ & \\
\hline \multirow{2}{*}{$p=\llbracket h \rrbracket$} & $h \mid l:$ & {$[l], \llbracket l \rrbracket$} & \\
& $h \nmid l:$ & $u(l, h), u(l, h) \wedge l$ & {$[h], \infty$} \\
\hline$p=\infty$ & & {$[l], \llbracket l \rrbracket$} & not applicable
\end{tabular}

It remains to show that there are no digons and no triangles in $\overline{\mathcal{A}}$. Of course, for two points in $P$ there is at most one joining line by (A2'). Every line in $L$ contains only one point not in $P$, and the only line not in $L$ through $\llbracket l \rrbracket$ is the 



$1 \frac{1}{1}=0$

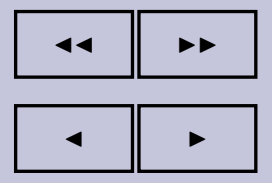
page $9 / 27$
go back
full screen
(a) The parallelisms $\mid$ and $\|$ are closed subsets of $L^{2}$.
(b) $\mathcal{A}$ is a stable quadrangle.
If $\mathcal{A}$ is stable (or topological), then the following assertions hold.
(c) The subsets $I \subseteq P \times L$ and $D_{1} \subseteq(P \cup L)^{2}$ and all panels are closed.
(d) The subsets $D_{3}$ and $D_{4}$ are open in $(P \cup \dot{\cup} L)^{2}$.
(e) The geometric operations $\vee$ and $\wedge$ are open and continuous maps.

close

Proof. (a) Choose $p \in P$, and define

$$
\alpha: L^{2} \rightarrow D_{1}(p)^{2},(g, h) \mapsto(\iota(p, g), \iota(p, h)) .
$$

Because $\mid$ is an equivalence relation by Lemma 2.4(c), we have that $g \mid h$ is equivalent to $\iota(p, g) \mid \iota(p, h)$, which is equivalent to $\iota(p, g)=\iota(p, h)$ by (A3), so $\mid=\alpha^{-1}\left(\operatorname{id}_{L}\right)$. This set is closed, because $L$ is a Hausdorff space and $\alpha$ is continuous.

Let $(g, h) \in \nVdash$. Since pencils have at least three elements, there is an $l \in L$ with $l \nmid g, h$. By what we have shown already $U:=\nmid(l)=\left\{l^{\prime} \in L: l^{\prime} \nmid l\right\}$ is open in $L$. Define

$$
\beta: U^{2} \rightarrow L^{2},\left(g^{\prime}, h^{\prime}\right) \mapsto(u(l, g), u(l, h)) .
$$

As above we have that $g^{\prime} \| h^{\prime}$ is equivalent to $u\left(l, g^{\prime}\right)=u\left(l, h^{\prime}\right)$ by Lemma 2.4(c) and (A4); so $\beta^{-1}\left(L^{2} \backslash \operatorname{id}_{L}\right)$ is an open neighbourhood of $(g, h)$ contained in $\nVdash$.

(b) For $(p, l) \in P \times L$ we have $d(p, l) \in\{1,5\}$ if and only if $\iota(p, l) \| l$; this fact is easily checked in a completion of $\mathcal{A}$. So $D_{3} \cap(P \times L)=\{(p, l) \in P \times L: \iota(p, l) \nVdash l\}$ is open by the continuity of $\iota$ and (a). Thus $D_{3}$ is open, because it is the union of this set and its inverse regarded as a relation. Thus $\mathcal{A}$ is a stable quadrangle, because the openness of the end-point map is here simply the continuity of $\pi$ and $\left.\lambda\right|_{D_{3}}$.

(c) This is a general fact about stable graphs whose vertex set is a Hausdorff space and whose diameter is bounded by the girth minus 2; see [17, Proposition 5.8]. If $\mathcal{A}$ is topological we can also proceed directly: the incidence relation $I=\{(p, l) \in P \times L: \iota(p, l)=l\}$ is closed, because $L$ is a Hausdorff space. The sets $\{p\} \times L$ and $P \times\{l\}$ are closed in $P \times L$; so panels are closed.

For (d) and (e) see Theorem 8.1(c) and (a).

Our next aim is to construct a compact completion of a topological affine quadrangle with locally compact point and line spaces. In order to define this notion, let $\mathcal{Q}=(P, L, I)$ be a topological generalized quadrangle; i.e. $P$ and $L$ 



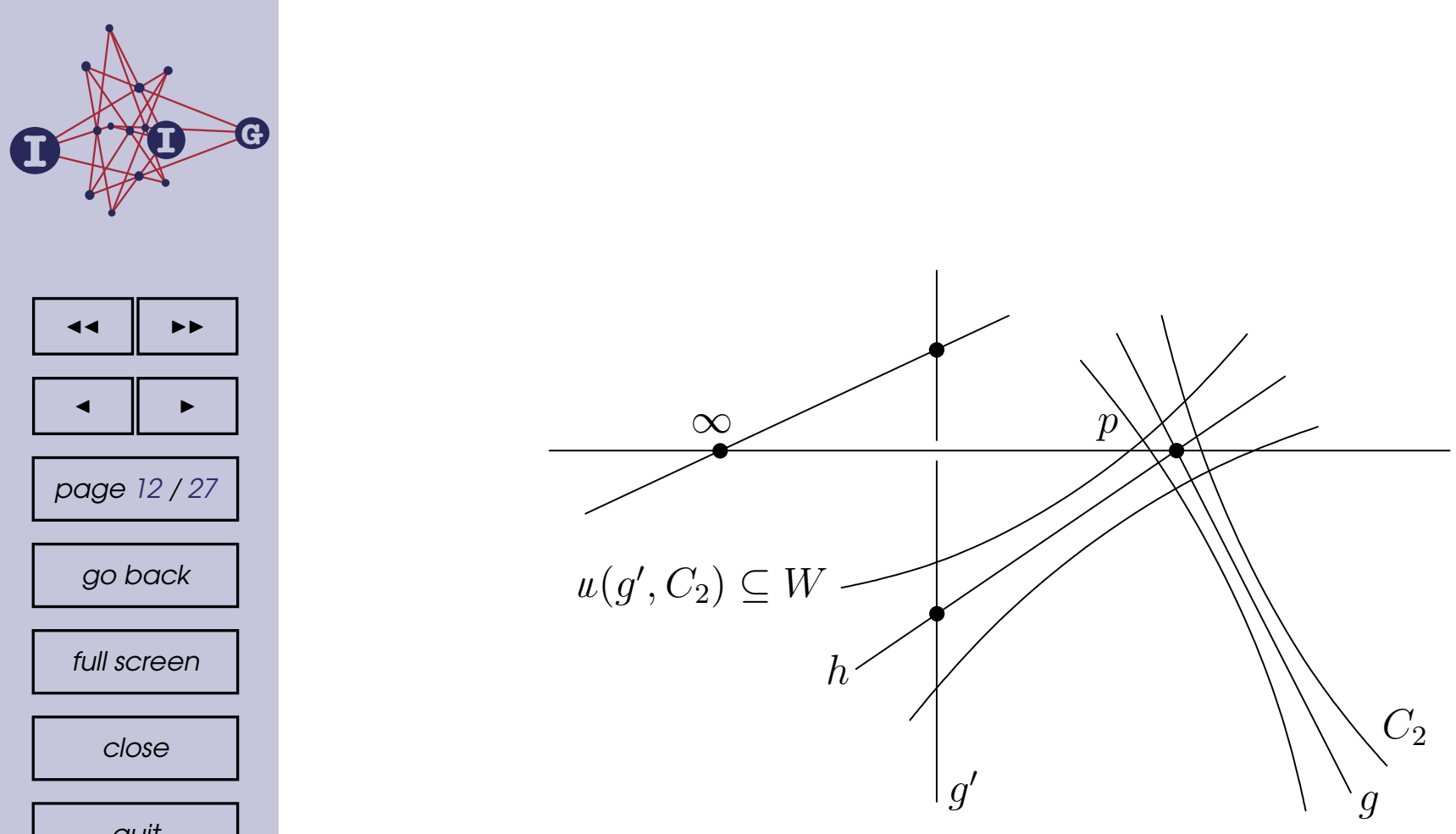

Figure 2: Local compactness of $P$

so $\wedge$ is defined on a closed subset of $C_{1} \times C_{2}$, and $C_{1} \wedge C_{2}$ is a compact neighbourhood of $g \wedge h \in C$ in $P_{\text {aff }}$, because $\wedge$ is continuous and open. Thus the compact set $C$ is covered by finitely many such sets, and $D_{1}(C)$ is compact by $\left(\mathrm{C}_{\gamma}\right)$, since $D_{1}\left(C_{1} \wedge C_{2}\right)$ is a closed subset of the compact set $D_{0,2}\left(C_{1}\right) \cap D_{0,2}\left(C_{2}\right)$ by Proposition 3.1(c).

(3) $L$ is compact: Let $\left(l_{\sigma}\right)$ be a net in $L$ without a cluster point in $L_{\text {aff }}$. Choose $q \in P_{\text {aff }}$. We have that finally $l_{\sigma} \notin D_{1}(q)$, because otherwise $\left(l_{\sigma}\right)$ would have a cluster point in the set $D_{1}(q) \subseteq L_{\text {aff }}$, which is compact by (2). Let $g$ be a cluster point of $\lambda\left(q, l_{\sigma}\right) \in D_{1}(q)$; see Figure 1 . By passing to a subnet $\left(l_{\sigma}\right)$ we can assume that $g$ is a limit. In order to show that $\left(l_{\sigma}\right)$ converges to $\lambda(\infty, g)$ let $h \in L_{\text {aff }} \cap D_{2}(\lambda(\infty, g))$ and let $U$ be an open neighbourhood of $h$ as well as $C$ a compact subset of $L_{\text {aff }}$. Choose $r \in P_{\text {aff }} \cap D_{1}(h)$. We show that $\lambda\left(r, l_{\sigma}\right)$ converges to $h$. Otherwise some cluster point $h^{\prime} \in D_{1}(r)$ of this net would not be parallel to $g$, because there are no triangles. Then $\left(l_{\sigma}\right)$ would have a cluster point in the compact set $D_{0,2}\left(C_{1}\right) \cap D_{0,2}\left(C_{2}\right) \subseteq L_{\text {aff }}$ for a compact neighbourhood $C_{1} \times C_{2} \subseteq \nmid$ of $\left(g, h^{\prime}\right)$, which we excluded. Thus $\lambda\left(r, l_{\sigma}\right)$ converges to $h$ and $\left(l_{\sigma}\right)$ is finally in $D_{2}(U) \backslash C$, because otherwise $\left(l_{\sigma}\right)$ would have a cluster point in $C \subseteq L_{\text {aff }}$.

(4) The sets $D_{1}(U) \backslash C$ form a neighbourhood basis of $p \in D_{2}(\infty)$ for neighbourhoods $U$ of a fixed $g \in D_{1}(p) \cap L_{\text {aff }}$ and compact subsets $C$ of $P_{\text {aff }}$ : Let $W$ be an open neighbourhood of $h \in D_{1}(p) \backslash\{g, p \vee \infty\}$ and $K \subseteq P_{\text {aff }}$ be compact; see Figure 2. Choose $g^{\prime} \in \nmid(g) \cap D_{2}(h)$. Then $d\left(g^{\prime}, g\right)=4$ and $u\left(g^{\prime}, g\right)=h$, so we can choose a compact neighbourhood $C_{2}$ of $g$ in $\nmid\left(g^{\prime}\right) \cap D_{4}\left(g^{\prime}\right)$ such that $u\left(g^{\prime}, C_{2}\right) \subseteq W$. Then $M:=D_{0,2}\left(g^{\prime}\right) \cap D_{0,2}\left(C_{2}\right) \subseteq D_{2}\left(C_{2}\right)$ is compact by $\left(\mathrm{C}_{\nmid}\right)$. We have $C_{2} \wedge M=D_{1}\left(C_{2}\right)$ and $D_{1}\left(C_{2}\right) \subseteq P_{\text {aff }} \cup D_{1}\left(u\left(g^{\prime}, C_{2}\right)\right) \subseteq P_{\text {aff }} \cup D_{1}(W)$. Since $C_{2}$ is compact and $W$ is open, the set $D_{1}\left(C_{2}\right) \backslash D_{1}(W) \subseteq P_{\text {aff }}$ is closed by 








\section{The Lie geometry of a Laguerre plane}

In this section we describe how affine quadrangles can be constructed from Laguerre planes. For the definitions of Laguerre planes and properties of topological Laguerre planes see the excellent overview in [23]. Let $\mathcal{L}=(S, C)$ be a Laguerre plane with point set $S$ and circle set $C$, and let $F:=\{(s, c) \in S \times C$ : $s \in c\}$ denote the set of flags of $\mathcal{L}$. The equivalence relation $\sim$ on $F$ of touching is defined by $(s, c) \sim(t, d)$ if and only if $s=t$ and either $c \cap d=\{s\}$ or $c=d$. The equivalence class of $(s, c)$ is denoted by $[s, c]$. Consider the following condition, which says that there is a unique circle in the class $[s, c]$ touching a given circle not containing $s$.

(B) For all $(s, c) \in F$ and $d \in C$ with $s \notin d$ there is a unique flag $(s, e) \in[s, c]$ such that $|e \cap d|=1$.

If condition (B) is satisfied, a function $\beta^{\mathrm{B}}(s, c, d):=e$ is defined. The condition is satisfied for finite Laguerre planes if the cardinality of the circles is even (see [13]) and for finite- and positive-dimensional locally compact topological Laguerre planes; see [20, 3.3].

Theorem 6.1. For a Laguerre plane $\mathcal{L}=(S, C)$ with flag set $F$ define the incidence geometry

$$
\mathcal{A}_{\mathcal{L}}:=(C, F / \sim, I) \quad \text { where } \quad I:=\{(c,[s, c]):(s, c) \in F\} .
$$

If condition (B) is satisfied, then $\mathcal{A}_{\mathcal{L}}$ is an affine quadrangle.

Proof. In order to prove that $\mathcal{A}_{\mathcal{L}}$ is an affine quadrangle we verify Condition (b) from Theorem 2.6 for the equivalence relations | and $\|$ on $L$ given by $[s, c] \mid[t, d]$ if and only if $s \| t$ (here $\|$ is the parallel relation in $\mathcal{L}$ ) and $[s, c] \|[t, d]$ if and only if $s=t$.

Now (A1) follows from the corresponding axiom for Laguerre planes, (A2') is just the fact that two distinct touching circles meet in only one point, (A3) follows from the axiom about parallel projection onto a circle, (A4) from the existence and uniqueness of touching circles and (A5) from condition (B).

Theorem 6.2. Let $\mathcal{L}$ be a topological Laguerre plane such that condition (B) is satisfied and $\beta^{\mathrm{B}}$ is continuous. Then $\mathcal{A}_{\mathcal{L}}$ is a topological affine quadrangle.

Proof. Let $q: F \rightarrow F / \sim$ be the quotient map. For an open subset $U$ of $F$ let $(s, c) \in q^{-1}(q(U))$. Then there is a flag $(s, d) \in U$ such that $(s, c) \sim(s, d)$. Choose $t \in d \backslash\{s\}$, and define the continuous map $f: F \rightarrow F,(s, c) \mapsto$ $(s, \beta(s, c, t))$. Then we have $f(s, c)=(s, d) \in U$ and $f^{-1}(U) \subseteq q^{-1}(q(U))$. Thus 





[12] M. Margraf, Topologische Laguerreräume und topologische verallgemeinerte Vierecke, Ph.D. Thesis, Kiel, 2001.

[13] S. E. Payne and J. A. Thas, Generalized quadrangles with symmetry, Simon Stevin 49 (1975/76), 3-32, 81-103.

[14] H. Pralle, Affine generalized quadrangles - an axiomatization, Geom. Dedicata 84 (2001), 1-23.

[15] N. Rosehr, Compactification of stable planes. Preprint.

[16] , Pseudo-isotopic contractions and compactness, Results Math. 44 (2003), 157-158.

[17] _ Stable Graphs and Polygons, Habilitationsschrift, Würzburg, 2003.

[18] H. Salzmann, D. Betten, T. Grundhöfer, H. Hähl, R. Löwen and M. Stroppel, Compact Projective Planes, de Gruyter, 1995.

[19] A. E. Schroth, Generalized quadrangles constructed from topological Laguerre planes, Geom. Dedicata 46 (1993), 339-361.

[20] _ Topological Circle Planes and Topological Quadrangles, Longman, 1995.

[21] G. Steinke, Eine Klassifikation 4-dimensionaler Laguerre-Ebenen mit großer Automorphismengruppe, Habilitationsschrift, Kiel, 1988.

[22] _ Semiclassical 4-dimensional Laguerre planes, Forum Math. 2 (1990), 233-247.

[23] _ Topological circle geometries, In: Handbook of incidence geometry, North-Holland (1995), 1325-1354

[24] B. Stroppel, Point-affine quadrangles, Note Mat. 20 (2000/01), 21-31.

[25] J. Tits, Sur la trialité et certains groupes qui s'en déduisent, Publ. Math. IHES 2 (1959), 14-60.

[26] H. Van Maldeghem, Generalized Polygons. Birkhäuser, 1998.

[27] D. Wagner, Ovale und ebene algebraische Kurven mit unendlicher Kollineationsgruppe, Ph.D. Thesis, Erlangen, 2004.

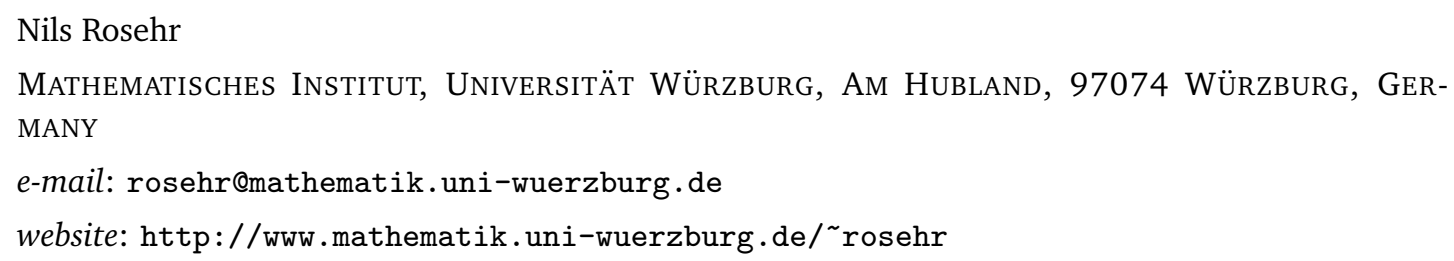

